\title{
Increased Synchronization of Cortical Oscillatory Activities between Human Supplementary Motor and Primary Sensorimotor Areas during Voluntary Movements
}

\author{
Shinji Ohara, ${ }^{1}$ Tatsuya Mima, ${ }^{1}$ Koichi Baba, ${ }^{4}$ Akio Ikeda, ${ }^{2}$ Takeharu Kunieda, ${ }^{3}$ Riki Matsumoto, ${ }^{1}$ \\ Junichi Yamamoto, ${ }^{1}$ Masao Matsuhashi, ${ }^{1}$ Takashi Nagamine, ${ }^{1}$ Kenichi Hirasawa, ${ }^{5}$ Tomokatsu Hori, ${ }^{5}$ \\ Tadahiro Mihara, ${ }^{4}$ Nobuo Hashimoto, ${ }^{3}$ Stephan Salenius, ${ }^{6}$ and Hiroshi Shibasaki ${ }^{1,2}$ \\ ${ }^{1}$ Human Brain Research Center and Departments of 2Neurology and ${ }^{3}$ Neurosurgery, Kyoto University Graduate School of \\ Medicine, Shogoin, Sakyo, Kyoto, 606-8507, Japan, 4The National Epilepsy Center, Shizuoka Higashi Hospital, \\ Urushiyama, Shizuoka, 420-8688, Japan, 5Department of Neurosurgery, Neurological Institute, Tokyo Women's Medical \\ University, Kawada-Cho, Shinjuku-Ku, Tokyo 162-8666, Japan, and 'Brain Research Unit, Low Temperature Laboratory, \\ Helsinki University of Technology, FIN-02015HUT, Espoo, Finland
}

In human, both primary and nonprimary motor areas are involved in the control of voluntary movements. However, the dynamics of functional coupling among different motor areas has not been fully clarified yet. Because it has been proposed that the functional coupling among cortical areas might be achieved by the synchronization of oscillatory activity, we investigated the electrocorticographic coherence between the supplementary motor and primary sensorimotor areas (SMA and S1-M1) by means of event-related partial coherence analysis in 11 intractable epilepsy patients. We found premovement increase of coherence between the SMA proper and S1-M1 at the frequency of $0-33 \mathrm{~Hz}$ and between the pre-SMA and S1-M1 at 0-18 Hz. Coherence between the SMA proper and M1 started to increase $0.9 \mathrm{sec}$ before the movement onset and peaked $0.3 \mathrm{sec}$ after the movement. There was no systematic difference within the SMA (SMA proper vs pre-SMA) or within the $S 1-M 1$, in terms of the time course as well as the peak value of coherence. The phase spectra revealed near-zero phase difference in $57 \%$ (20 of 35 ) of region pairs analyzed, and the remaining pairs showed inconsistent results. This increase of synchronization between multiple motor areas in the preparation and execution of voluntary movements may reflect the multiregional functional interactions in human motor behavior.

Key words: event-related coherence; primary sensorimotor area; supplementary motor area; electrocorticography; voluntary movements; functional coupling
In the motor system, the primary motor (M1) and the nonprimary motor areas, including the supplementary motor area (SMA), are both active during voluntary movements (Tanji and Kurata, 1982; Ikeda et al., 1992; Shibasaki et al., 1993; Deiber et al., 1996). Furthermore, anatomical studies show dense direct connections among those motor areas (Dum and Strick, 1991; Luppino et al., 1993). It is conceivable, therefore, that multiple motor areas are functionally bound together to work as a global network in performing a motor act. One possible advantage of the networktype system may be the adaptability against the regional dysfunction. Motor deficits caused by localized cortical lesions often show dramatic recovery. Imaging studies using hand movement tasks showed that, in patients with ischemic lesions in M1 showing good motor recovery, activation of the premotor areas, including the SMA, was increased (Cramer et al., 1997; Seitz et al., 1998). Furthermore, motor deficits caused by the SMA lesions are known to be transient, possibly because of compensatory process

Received May 2, 2001; revised Sept. 6, 2001; accepted Sept. 7, 2001.

This work was supported by Grants-in-Aid for Scientific Research on Priority Areas 08279106, Scientific Research (C) 10670583, (C) 1167621, and (C) 12210012 from Japan Ministry of Education, Science, Sports, and Culture, Research for the Future Program from the Japan Society for the Promotion of Science Grant JSPS-RFTF97L00201, and Grant-in-Aid for Encouragement of Young Scientists 13780634 from the Japan Society for the Promotion of Science.

Correspondence should be addressed to Dr. Hiroshi Shibasaki, Human Brain Research Center and Department of Neurology, Kyoto University Graduate School of Medicine, Shogoin, Sakyo, Kyoto, 606-8507, Japan. E-mail: shib@kuhp. kyoto-u.ac.jp.

Copyright (ㄷ) 2001 Society for Neuroscience $\quad 0270-6474 / 01 / 219377-10 \$ 15.00 / 0$ within the motor system (Laplane et al., 1977; Rostomily et al., 1991). Therefore, the functionally coupled cortical network might be a physiological substrate of large-scale motor plasticity.

Oscillations of cortical neuronal activity and local field potential are recorded in association with various brain functions such as visual (Eckhorn et al., 1988; Gray and Singer, 1989), olfactory (Freeman, 1972), auditory (Pantev et al., 1991), and sensorimotor systems (Pfurtscheller and Aranibar 1979; Sanes and Donoghue, 1993; Murthy and Fetz, 1996; Donoghue et al., 1998). Recently, it is proposed that synchronous oscillations in two brain areas become coupled each other in binding the features separately processed in each of two areas (Singer, 1993; Singer and Gray, 1995).

Direct cortical recording in humans shows that voluntary movements modulate oscillatory activities arising from M1 and the SMA proper (Toro et al., 1995; Crone et al., 1999a,b; Ohara et al., 2000a). The premovement decrease of oscillatory activities is believed to indicate the activated state of the underlying cortex (Pfurtscheller, 1992). Recent studies of cortico-muscular coherence demonstrated functional coupling between motor areas and spinal motoneurons, indicating a significant role of cortical oscillatory activity in the motor control (Murthy and Fetz, 1992; Conway et al., 1995; Salenius et al., 1996, 1997; Brown et al., 1998; Halliday et al., 1998; Mima and Hallett 1999; Mima et al., 1999, 2000; Ohara et al., 2000b).

Attempts have been made to demonstrate a correlation among oscillations arising from multiple motor areas such as M1 and 
Table 1. Clinical profiles of the patient studies

\begin{tabular}{|c|c|c|c|c|c|c|c|c|c|c|c|}
\hline Patient & 1 & 2 & 3 & 4 & 5 & 6 & 7 & 8 & 9 & 10 & 11 \\
\hline Age/sex & $28 / \mathrm{M}$ & $13 / \mathrm{F}$ & $34 / F$ & $32 / \mathrm{F}$ & $23 / \mathrm{F}$ & $31 / \mathrm{M}$ & $24 / F$ & $15 / \mathrm{M}$ & $50 / \mathrm{M}$ & $34 / \mathrm{M}$ & $31 / F$ \\
\hline Diagnosis & Lt FLE & Lt FLE & Rt FLE & Lt FLE & Lt FLE & Rt FLE & Rt FLE & Lt FLE & $\begin{array}{l}\text { Tumor } \\
\text { Lt frontal }\end{array}$ & $\begin{array}{l}\text { Tumor } \\
\text { Rt frontal }\end{array}$ & Lt FLE \\
\hline Drugs & $\begin{array}{c}\text { PHT PB } \\
\text { CBZ ZNS } \\
\text { VPA }\end{array}$ & $\begin{array}{c}\text { CBZ PHT } \\
\text { ZNS PB }\end{array}$ & $\begin{array}{c}\text { PHT PB } \\
\text { PRM CBZ } \\
\text { ZNS VPA } \\
\text { CZP NZP }\end{array}$ & $\begin{array}{c}\text { PHT CBZ } \\
\text { ZNS PB }\end{array}$ & $\begin{array}{c}\text { PHT CBZ } \\
\text { ZNS }\end{array}$ & $\begin{array}{c}\text { PB VPA } \\
\text { ZNS }\end{array}$ & PHT CBZ & $\begin{array}{c}\text { PHT PRM } \\
\text { CBZ }\end{array}$ & & & $\begin{array}{c}\text { PHT CBZ } \\
\text { PB }\end{array}$ \\
\hline
\end{tabular}

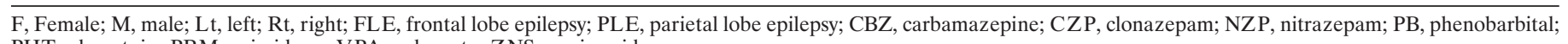
PHT, phenytoin; PRM, primidone; VPA, valproate; ZNS, zonisamide.

SMA (Rappelsberger et al., 1994; Andrew and Pfurtscheller, 1995; Leocani et al., 1997; Gerloff et al., 1998; Andres et al., 1999). However, the scalp-recorded electroencephalogram (EEG) has a limited spatial resolution because of volume conduction of electric activity, In this regard, the electrocorticogram (ECoG) is expected to provide us with a valuable information. Therefore, to test our hypothesis that not only the local cortical activation but also the inter-regional functional coupling may be modulated by motor tasks, we analyzed movement-related change of ECoG coherence among different motor areas.

\section{MATERIALS AND METHODS}

Subjects. We studied 11 right-handed patients (six females and five males, age 13-50 years) with medically intractable partial epilepsy or brain tumor (Table 1). All these patients underwent chronic implantation of subdural electrodes for the purpose of surgical treatment. Cortical electric potentials were recorded with platinum electrodes (Ad-Tech Company, Racine, WI). Each electrode was $3 \mathrm{~mm}$ in diameter, and the center-to-center interelectrode distance was $1 \mathrm{~cm}$. The electrodes were placed at both the mesial and lateral surface of the frontoparietal lobes on the left hemisphere in seven subjects (patients $1,2,4,5,8,9$, and 11) and on the right in four subjects (patients $3,6,7$, and 10). This invasive technique was performed to identify the epileptogenic area by recording epileptic discharges and to delineate the function of the cortical areas around the epileptogenic site by cortical electric stimulation and recording of somatosensory evoked potentials (SEPs). Written informed consent was obtained from all subjects after the purpose, and possible consequences of the studies were explained (Clinical Research protocol number 79 approved by the Committee of Medical Ethics, Graduate School of Medicine, Kyoto University, for patient 11 and protocol number 98-1 approved by the Ethics Committee of the National Epilepsy Center, Shizuoka, for patients 1-8). In two subjects (patients 9 and 10), oral informed consent was obtained at Tokyo Women's Medical University. Other neurophysiological findings of patients 3,4 , and 11 were reported elsewhere for entirely different purposes (Ikeda et al., 1999a,b; Kunieda et al., 2000; Ohara et al., 2000a).

Cortical functional mapping. Electric stimulation was performed by delivering electric current to each electrode. Details of the stimulation method have been described elsewhere (Lüders et al., 1987a; Ikeda et al., 1992; Lesser et al., 1992). Cortical sites where the stimulation elicited muscle contraction were defined as "positive motor areas", and the areas where stimulation interfered with tonic muscle contraction or rapid alternating movements were defined as "negative motor areas" (Lüders et al., 1987b, 1992). For recording SEPs, electric stimulation of the median nerve contralateral to the side of electrode implantation was performed at the wrist with a stimulus intensity of $20 \%$ above the motor threshold for the abductor pollicis brevis muscle.

Identification of primary somatosensory area (S1) and M1 was based on subjective sensation and positive motor responses, respectively, elicited by electric stimulation of each electrode. Because the electrodes were located on the crown of the gyrus, M1 in the present study might include both Brodmann's areas 4 and 6 (Zilles et al., 1995; White et al., 1997). The central sulcus was identified based on the distribution of N20-P20 deflection of SEPs in four subjects (patients 1, 3, 5 and 11) (Allison et al., 1991). In five subjects (patients 2, 4, 6, 9, and 10), the anatomical configuration judged by three-dimensional (3-D) magnetic resonance images (MRIs) taken after implantation of electrode grids was used for its determination. In the mesial cortex, the SMA proper was identified by its unique response to stimulation, consisting of predominantly tonic motor response of the upper as well as lower limbs, either unilaterally or bilaterally, and of trunk, neck, and face (Fried et al., 1991; Lim et al., 1994). When no positive motor responses were elicited, the electrodes located posterior to the vertical anterior commissural (VAC) line on the mesial surface of the superior frontal gyrus were judged to be on the SMA proper (Picard and Strick, 1996; Wise et al., 1996; Zilles et al., 1996). The somatotopy in the SMA proper was determined by taking the distribution of the movement-related cortical potential (MRCP) into account (Ohara et al., 2000a). Anatomical location of the VAC line was determined based on the skull x-ray film and T1-weighted MRI in nine subjects (except for patients 1 and 7). Namely, the lateral view of the former was obtained after implantation of subdural electrodes and superimposed on the midsagittal plane of the latter. This enabled us to visualize the exact anatomical location of subdural electrodes (Ikeda et al., 1995, 1996). Those electrodes that were located just rostral to the VAC line or showed negative motor response to cortical stimulation were judged to be on the pre-SMA regardless of their location.

In the subjects whose anatomical configuration of sulci was identified by 3-D MRIs (patients 1,2, 6, 9, and 10) or visual inspection during surgery (patient 11), the precentral sulcus was also determined.

Motor task. The subjects lay supine on a bed with the arm contralateral to the implanted electrodes placed on a pillow. They performed a brisk, voluntary extension of the middle finger (patients 1-10) or the wrist (patient 11) at a self-paced rate of once per 6-8 sec. They were trained before the recording so that they could move their finger or wrist briskly with a sufficiently long intertrial interval. The motor performance was continuously monitored with on-line electromyogram (EMG) recorded from the extensor digitorum communis (EDC) muscle for the middle finger extension and the extensor carpi radialis (ECR) muscle for the wrist extension.

Data acquisition. ECoGs from 28-32 subdural electrodes and EMGs were continuously recorded. All subdural electrodes were referenced to a scalp electrode placed on the mastoid process contralateral to the side of electrode implantation. EMG from the EDC or ECR muscle was recorded by a pair of cup electrodes. The bandpass filter for data acquisition was set to $0.016-120 \mathrm{~Hz}$ for both ECoG and EMG. All input signals were digitized at a sampling rate of $500 \mathrm{~Hz}$ and stored on magneto-optical disks with a digital EEG equipment (EEG2100; Nihon kohden, Tokyo, Japan). Data recorded from the electrodes either placed on the lesions demonstrated by MRI or showing epileptic discharges were excluded from further analysis.

Analysis. The EMG onset of each finger or wrist movement was visually determined on the continuously recorded data off-line. Trials containing artifacts or incomplete relaxation between movements were excluded from analysis. A total of 51-200 trials were selected for averaging for each subject.

Estimates of auto and coherence spectra were calculated by a fast Fourier transform algorithm implemented on Matlab 5.3 (Mathworks, Natick, MA). An estimate of coherence between ECoG signals recorded with reference to a common electrode might show an apparent increase of coherence as the result of introduction of activities from the reference electrode commonly into the two exploring electrodes (Andrew and Pfurtscheller, 1996). In the present study, we used partial instead of ordinary coherence to solve this reference problem (Mima et al., 2000). Partial coherence was computed by adopting the ECoG signal from a 

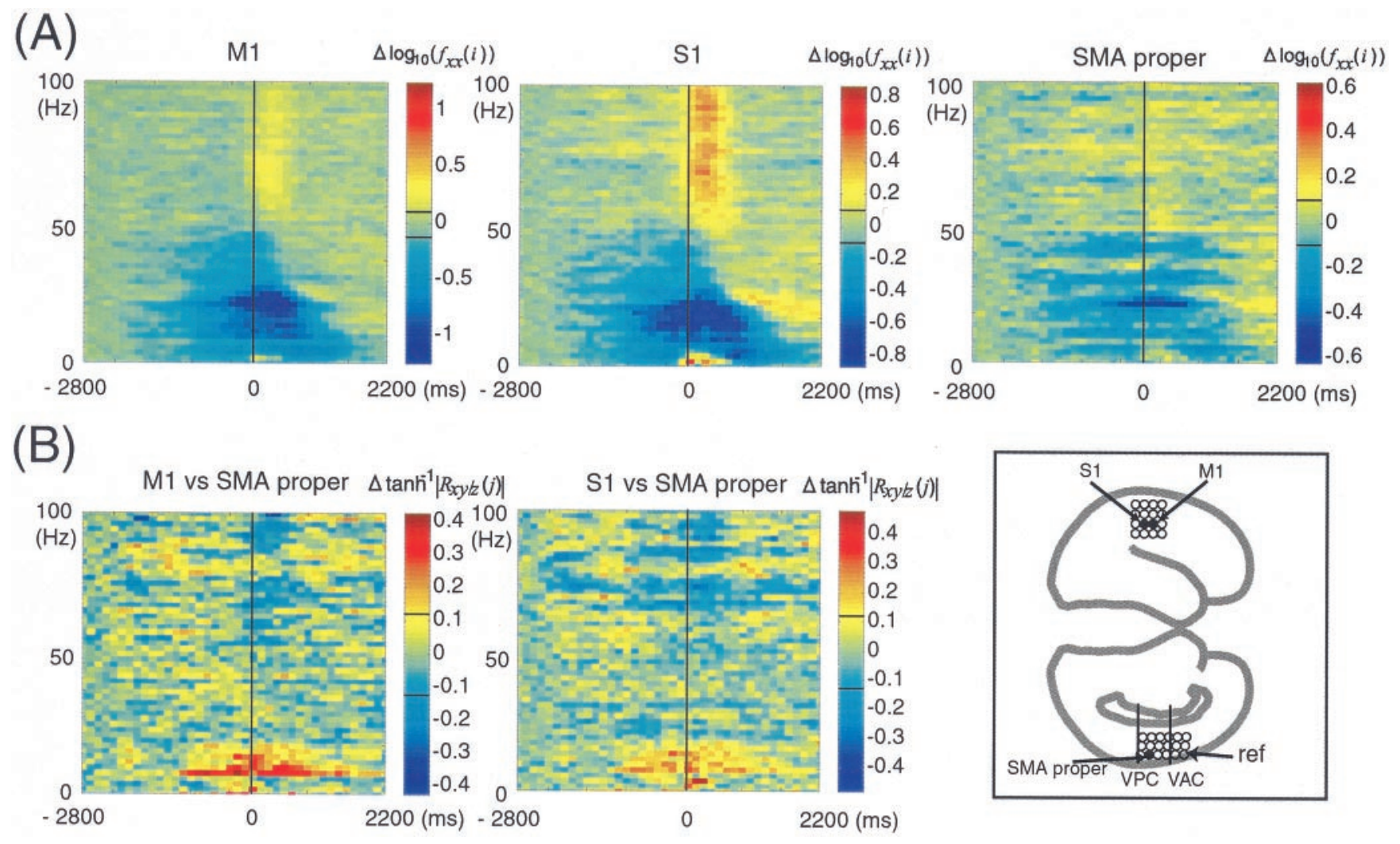

Figure 1. Time-frequency map for power change $(A)$ and for coherence change $(B)$ in patient 3. In the diagram, the anatomical locations of electrodes corresponding to M1, S1, and the SMA proper are schematically shown. VAC and VPC indicate vertical anterior and posterior commissural line, respectively. The electrode used as $Z$ in the equation for calculation of partial coherence is also shown (ref). Note clear ERD pattern in M1, S1, and the SMA proper in the frequency range $<50 \mathrm{~Hz}$, followed by MPowI. Significant MPowI in $>60 \mathrm{~Hz}$ was observed in S1 and M1, but not in the SMA proper. Significant increase in coherence was demonstrated for M1 versus the SMA proper and S1 versus the SMA proper in the frequency range of 10-20 Hz, with the peak occurring around the time of movement onset. The horizontal bars in color scale indicate the $95 \%$ confidence limits.

presumably most irrelevant electrode in each subject, which was far away from functional electrodes judged from the functional mapping and was devoid of significant MRCP.

Partial coherence was calculated as follows (Halliday et al., 1995; Mima et al., 2000):

$$
\left|R_{x y / z}(j)\right|^{2}=\frac{\left|f_{x y}(j)-\frac{f_{x z}(j) f_{z y}(j)}{f_{z z}(j)}\right|^{2}}{\left(f_{x x}(j)-\frac{\left|f_{x z}(j)\right|^{2}}{f_{z z}(j)}\right)\left(f_{y y}(j)-\frac{\left|f_{z y}(j)\right|^{2}}{f_{z z}(j)}\right)}
$$

where coherence was expressed as:

$$
\left|R_{x y}(j)\right|^{2}=\frac{\left|f_{x y}(j)\right|^{2}}{f_{x x}(j) f_{y y}(j)} .
$$

In this equation, $f_{x x}(i), f_{y y}(i)$, and $f_{z z}(i)$ indicate estimates of autospectra of the ECoG signals, $X, Y$, and $Z$ for a given frequency of $(i)$, and $f_{x y}(i)$, $f_{x z}(i)$, and $f_{x y}(i)$ denote cross-spectra between each pair of signals. For a statistical comparison, normalized coherence, which denotes the arctanh-transformed value of the square root of partial coherence, was computed (Halliday et al., 1995). The ECoG power was normalized on a logarithmic scale. To obtain a time course of partial coherence and power, a $512 \mathrm{msec}$ window was shifted from $3072 \mathrm{msec}$ before to 2048 msec after the movement onset in steps of $128 \mathrm{msec}$, making 40 epochs for each trial. Therefore, the center of the moving window was shifted from $2.8 \mathrm{sec}$ before to $2.2 \mathrm{sec}$ after the movement onset.

The baseline period was defined as the segment from 2.8 to $2.3 \mathrm{sec}$ (initial 5 epochs) before the EMG onset. The mean \pm 2 SD of normalized coherence and that of power values during the baseline period were defined as the baseline values. The frequency bands of
$0-6 \mathrm{~Hz}(\delta-\theta), 8-12 \mathrm{~Hz}(\alpha), 14-18 \mathrm{~Hz}(\beta 1), 20-23 \mathrm{~Hz}(\beta 2), 25-33 \mathrm{~Hz}$, $35-41 \mathrm{~Hz}$, and up to $100 \mathrm{~Hz}$ in steps of $\sim 10 \mathrm{~Hz}$ were analyzed. The peak time was defined as the time of the maximal increase in coherence and that of the maximal decrease in power in a given frequency band. The onset time was evaluated by using the regression line that started when the signal exceeded the baseline value and ended at the peak (Nagamine et al., 1996). The times of peak and onset and the value at the peak time were determined in the frequency band showing the maximal change in coherence or power. Thus, the frequency band, where the peak and onset were estimated, was variable across pairsof-channels for coherence and channels for power. Furthermore, to investigate the spatial distribution of coherence computed relative to one electrode and that of power decrease, we made distribution maps showing the peak coherence value and the maximal power decrease for each frequency band. Because the peak value of coherence and the maximal power decrease were determined at each channel, their times were not necessarily identical among electrodes. In a preliminary analysis, we constructed the distribution maps of coherence and power at the same peak time with respect to the EMG onset, which showed essentially the same spatial pattern.

To measure the temporal relation between two ECoG signals, phase spectrum, $\Phi_{x y / z}(j)$, was defined as the argument of the cross-spectrum as follows,

$$
\Phi_{x y / z}=\arg \left\{f_{x y}(j)-\frac{f_{x z}(j) f_{z y}(j)}{f_{z z}(j)}\right\} .
$$

$\Phi_{x y / z}(j)$, has a valid interpretation only when significant correlation is present between two ECoG signals (Halliday et al., 1995). Phase information was analyzed at the time of the coherence peak only in the frequency band where coherence showed a significant increase. The $95 \%$ confidence limits of phase were defined as follows, 
Figure 2. Power in M1 and the SMA proper $(A)$ and coherence $(B)$ spectra in patient 3 . A thin line indicates spectra calculated during the baseline period (from 2800 to $2300 \mathrm{msec}$ before the movement), and a thick line those around the movement onset (from $300 \mathrm{msec}$ before to $300 \mathrm{msec}$ after the movement onset). Power suppression in the frequency range $<30-40 \mathrm{~Hz}$ was observed in both M1 and the SMA proper during movement. Coherence between the two areas increased at $\sim 10 \mathrm{~Hz}$. Interrupted horizontal lines in $A$ indicate the frequency bands where the difference between two power spectra exceeds the $95 \%$ confidence limit. A dotted horizontal line in $B$ indicates the $95 \%$ confidence limit for coherence.

$$
\Phi_{x y / z}(j) \pm 1.96\left\{\frac{1}{2 L}\left(\frac{1}{\left|R_{x y / z}(j)\right|^{2}}-1\right)\right\}^{1 / 2}
$$

(A)

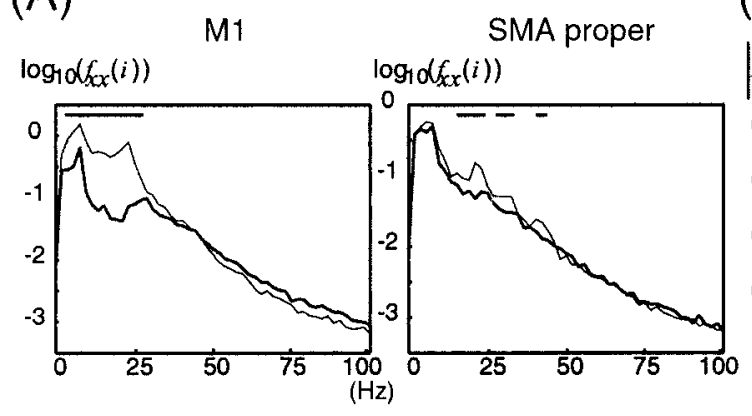

(B)

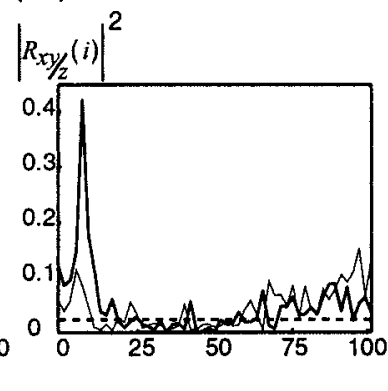

If this range includes zero in a given frequency, it is defined that the phase lag equals near-zero.

A Student's $t$ test and the Mann-Whitney $U$ test were used for statistical comparisons of coherence value and onset-peak times, respectively. The peak value of coherence was further compared with twofactor factorial ANOVAs (mesial $\times$ lateral; $2 \times 2$ ).

\section{RESULTS}

Hereafter, M1, S1, and the SMA proper denote specifically the hand area of each region unless otherwise designated. The electrodes corresponding to M1 were identified in all the subjects and those to S1 in all but one subject (patient 9). The SMA proper was identified by cortical stimulation in seven subjects (patients 1 , 2, and 5-9) and based on the anatomical location and MRCP findings in the remaining four subjects (patients 3, 4, 10, and 11). As far as the pre-SMA was concerned, a negative response to cortical stimulation was obtained in two subjects (patients 2 and 7 ), and it was anatomically defined in five subjects (patients 3, 4, 5, 6, and 11).

\section{Power change related to movements}

The movement-related change of power was analyzed in each of the four areas (Figs. $1 A, 2,3 A$ ). The results in three subjects (patients 3, 4, and 11) for S1, M1, and the SMA proper were reported in the previous paper (Ohara et al., 2000), and the findings were consistent among all other subjects analyzed in the present study. In M1 and S1, the decrease of power (movementrelated power decrease; MPowD) occurred $1.3 \pm 0.6$ and $1.2 \pm$ $0.6 \mathrm{sec}$ before the movement onset, respectively (Table 2). It occurred in the frequency range below $\sim 55 \mathrm{~Hz}$ (Fig. 1A). The increase of power after the movement (movement-related power increase; MPowI) was observed in the $\beta$ and $\gamma$ bands. The MPowI followed the MPowD in the $\beta$ and low $\gamma$ bands $(\sim 10-30 \mathrm{~Hz})$, whereas in the high $\gamma$ band $(>55 \mathrm{~Hz})$, the MPowI occurred just around the movement onset (Fig. $1 A$ ). In the SMA proper, both the MPowD and the following MPowI were observed in the frequency range of $<50 \mathrm{~Hz}$ (Fig. $1 A$ ). The MPowD in the SMA proper started at $1.8 \pm 0.8 \mathrm{sec}$ before the movement (Table 2). $\gamma$ band MPowI in the SMA proper, however, was present only in two subjects (patients 5 and 9). As for the pre-SMA, a clear MPowD pattern was observed in five subjects (patients 2, 3, 5, 7, and 11) in the frequency range of $<35 \mathrm{~Hz}$ (data not shown). No MPowI was observed in the pre-SMA.

The maximal MPowD was most frequently seen in the $\beta$ band ( $\beta 1$ and $\beta 2)(71 \% ; 22$ of 31$)$ in all areas except for the pre-SMA, where MPowD of a relatively wide frequency distribution was observed.

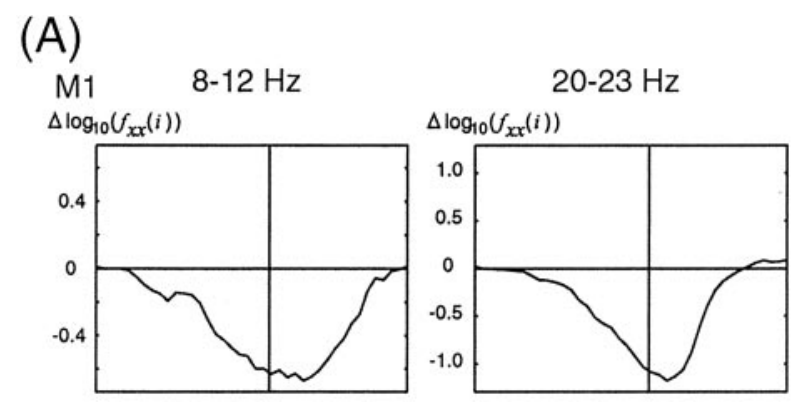

SMA proper
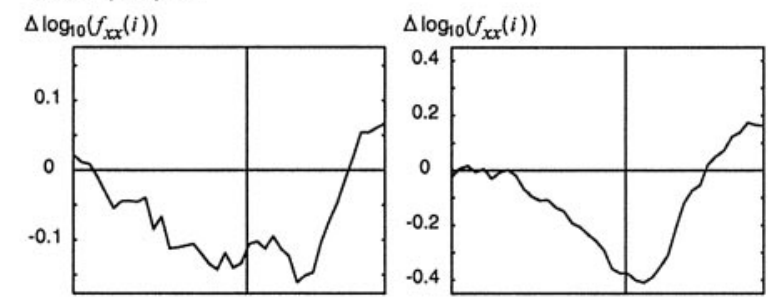

(B)

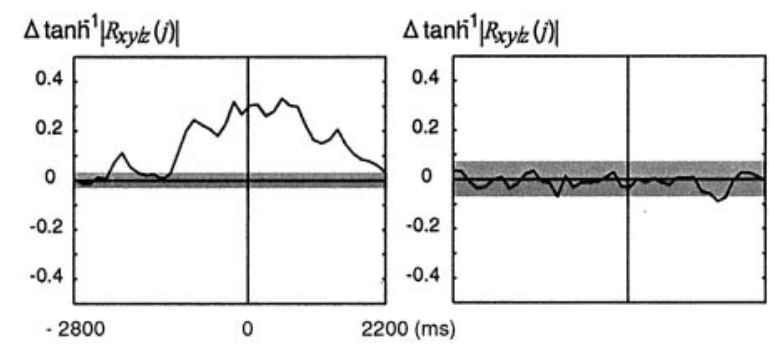

Figure 3. Time course of power $(A)$ and coherence $(B)$ in the frequency of $8-12$ and $20-23 \mathrm{~Hz}$. Both M1 and the SMA proper showed an MPowD pattern in both frequency bands, whereas significant coherence increase was present only in $8-12 \mathrm{~Hz}$, which started $\sim 1.4 \mathrm{sec}$ before the movement onset. Shaded areas indicate the baseline value.

\section{Functional coupling among different motor areas}

Table 3 shows the coherence data including onset and peak times, peak value, and frequency bands in each of region pairs for each individual subject.

\section{SMA proper versus S1-M1}

Figure 2 shows the coherence and power spectra around the movement onset and during the baseline period in patient 3 . Coherence between the SMA proper and M1 showed a transient increase with a peak occurring around the time of the movement onset (Figs. $1 B, 3 B$ ). A significant increase in coherence between the SMA proper and M1 was observed in 10 subjects (except for 


\begin{tabular}{|c|c|c|c|}
\hline & \multicolumn{2}{|l|}{$\underline{\text { Time }(\mathrm{sec})^{a}}$} & \multirow{2}{*}{$\begin{array}{l}\text { Peak value }{ }^{b} \\
(\text { Mean } \pm \mathrm{SD})\end{array}$} \\
\hline & Onset & Peak & \\
\hline \multicolumn{4}{|l|}{ Power } \\
\hline M1 & $-1.3 \pm 0.6$ & $+0.3 \pm 0.1$ & $-0.64 \pm 0.30$ \\
\hline $\mathrm{S} 1$ & $-1.2 \pm 0.6$ & $+0.4 \pm 0.2$ & $-0.61 \pm 0.20$ \\
\hline SMA proper & $-1.8 \pm 0.8$ & $+0.4 \pm 0.2$ & $-0.30 \pm 0.12$ \\
\hline Pre-SMA & $-1.3 \pm 1.1$ & $+0.5 \pm 0.3$ & $\begin{array}{c}-0.20 \pm 0.07 \\
\left(\log _{10}\left(\mu V^{2}\right)\right)\end{array}$ \\
\hline \multicolumn{4}{|l|}{ Coherence } \\
\hline \multicolumn{4}{|c|}{ SMA proper versus } \\
\hline M1 & $-0.9 \pm 0.8$ & $+0.3 \pm 0.3$ & $0.31 \pm 0.10$ \\
\hline $\mathrm{S} 1$ & $-0.6 \pm 0.7$ & $+0.3 \pm 0.3$ & $0.31 \pm 0.08$ \\
\hline \multicolumn{4}{|c|}{ Pre-SMA versus } \\
\hline M1 & $-0.7 \pm 0.4$ & $+0.4 \pm 0.2$ & $0.26 \pm 0.05$ \\
\hline $\mathrm{S} 1$ & $-0.2 \pm 0.3$ & $+0.4 \pm 0.4$ & $0.24 \pm 0.09$ \\
\hline M1 versus S1 & $-0.4 \pm 0.8$ & $+0.3 \pm 0.3$ & $0.33 \pm 0.08$ \\
\hline
\end{tabular}

${ }^{a}$ Expressed as the interval before (indicated by - ) or after (indicated by + ) the movement onset.

${ }^{b}$ Values at the time of peak.

patient 11) and between the SMA proper and S1 in eight subjects (except for patients 4, 6, and 9). The upper limits of frequency showing significant coherence increase varied among subjects, ranging from 12 to $33 \mathrm{~Hz}$. Even when the data were re-analyzed with a narrower analysis window of $128 \mathrm{msec}$ to detect a very transient coherence change, no significant coherence increase was observed in the frequency range of $>30 \mathrm{~Hz}$ except for patient 11 . In $50 \%$ of the subjects (four subjects for M1 vs the SMA proper and five for S1 vs the SMA proper), coherence increase was maximal in the $\alpha$ band, and only two subjects showed the largest coherence increase in the $\beta$ band ( $\beta 2)$. Coherence with the SMA proper was not significantly different between M1 and S1 either in the peak value ( $p=0.94 ; t$ test) or the time epochs of peak and onset ( $p=0.76$ and 0.42 ; Mann-Whitney $U$ test). The onset of coherence increase between the SMA proper and M1 and between the SMA proper and S1 was significantly later than that of MPowD in the SMA proper ( $p=0.019$ and 0.006; MannWhitney $U$ test) (Table 2). It tended to be later than that of MPowD in M1 and S1 ( $p=0.14$ and 0.07; Mann-Whitney $U$ test).

Figure 4 shows the spatial distribution of significant coherence increase computed relative to the SMA proper over the lateral surface in patient 6 . In this subject, significant coherence increase occurred in the frequency range of $\sim 23 \mathrm{~Hz}$. Maximal coherence increase was present at the postcentral area in the $\alpha$ band and over the precentral area in the $\beta 2$ band. The spatial relation between MPowD and coherence was variable among subjects. Significant coherence also occurred in the gyrus just rostral to the precentral sulcus in four of six subjects whose precentral sulcus was identified (patients 2, 6, 9, and 10).

Among 10 subjects showing significant coherence increase between the SMA proper and M1, seven (patients 1-7) had nearzero phase. The remaining three showed inconsistent results. The phase difference between the SMA proper and M1 in the frequency band showing maximal coherence increase was $2 \pm 7^{\circ}$. For the phase difference between the SMA proper and S1, five of eight subjects (patients 1,5, 7, 8, and 11) revealed near-zero lag relation $\left(-1 \pm 8^{\circ}\right)$, whereas the remaining three showed inconsistent results.

\section{Pre-SMA versus S1-M1}

Significant coherence increase between the pre-SMA and M1 was observed in five subjects (patients 2, 3, 4, 5, and 11) and between the pre-SMA and S1 in four subjects (patients 2, 3, 5, and 11). The upper limit of frequency showing significant coherence increase varied among subjects, ranging from 6 to $18 \mathrm{~Hz}$. Re-analysis with a more narrow window of $128 \mathrm{msec}$ did not change the results. No subject showed a maximal coherence increase for the frequencies higher than the $\beta$ range. Coherence with the pre-SMA was not significantly different between M1 and S1 either in the peak value either in peak value ( $p=0.72 ; t$ test) or time of peak or onset ( $p>0.99$ and $p=0.09$, respectively; Mann-Whitney $U$ test) (Table 2). No comparison with power change for onset time was done because of the small number of subjects.

Maximal coherence increase computed relative to the preSMA was located in the precentral area in the frequency range of $\sim 23 \mathrm{~Hz}$. The location of maximal MPowD moved across the central sulcus as a function of frequency in patient 3 (Fig. 5). However, the spatial relation between maximal coherence increase and MPowD was not consistent among subjects.

Phase analysis revealed near-zero phase difference between the pre-SMA and M1 in three of five subjects (patients 3-5). The phase difference in the frequency band showing maximal coherence increase was $2 \pm 2^{\circ}$. The remaining subjects showed inconsistent results. The phase difference between the pre-SMA and S1 showed inconsistent results in all subjects because of variability in terms of frequency range.

\section{M1 versus $\mathbf{S 1}$}

Among 10 subjects whose M1 and S1 were identified, seven showed a significant coherence increase (patients 1, 2, 5, 7, 8, 10, and 11) (Fig. 6). The time course of coherence was not significantly different from that between mesial (the SMA proper or the pre-SMA) and lateral (S1 or M1) cortices (Table 2). The upper limit of frequency showing significant coherence increase ranged from 6 to $41 \mathrm{~Hz}$. No significant coherence was observed in the high $\gamma$ band $(>60 \mathrm{~Hz})$, even with a more narrow analysis window of $128 \mathrm{msec}$. The largest increase of coherence was present in the 


\begin{tabular}{|c|c|c|c|c|c|c|c|c|}
\hline \multirow[b]{2}{*}{$\mathrm{Pt}$} & \multicolumn{4}{|c|}{ SMA proper versus M1 } & \multicolumn{4}{|c|}{ SMA proper versus S1 } \\
\hline & Freq $(\mathrm{Hz})$ & Onset (sec) & Peak (sec) & Peak value & Freq & Onset & Peak & Peak value \\
\hline 1 & $\sim 12$ & -0.4 & 0.3 & 0.27 & $\sim 23$ & -0.6 & 0.4 & 0.27 \\
\hline 2 & $\sim 12$ & 0.0 & 0.6 & 0.22 & $\sim 12$ & -0.1 & 0.4 & 0.43 \\
\hline 3 & $\sim 18$ & -2.3 & 0.5 & 0.33 & $\sim 18$ & -2.0 & 0.0 & 0.29 \\
\hline 4 & $\sim 33$ & -1.9 & 0.3 & 0.29 & NS & NS & NS & NS \\
\hline 5 & $\sim 12$ & 0.1 & 0.5 & 0.51 & $\sim 18$ & -0.1 & 0.5 & 0.44 \\
\hline 6 & $\sim 23$ & -0.5 & 0.1 & 0.22 & NS & NS & NS & NS \\
\hline 7 & $\sim 18$ & -1.3 & -0.3 & 0.24 & $\sim 18$ & -0.4 & -0.3 & 0.19 \\
\hline 8 & $\sim 23$ & -0.9 & -0.4 & 0.45 & $\sim 23$ & -1.2 & 0.4 & 0.28 \\
\hline 9 & $\sim 18$ & -1.0 & 0.5 & 0.29 & - & - & - & - \\
\hline 10 & $\sim 18$ & -0.5 & 0.3 & 0.28 & $\sim 12$ & -0.1 & 0.5 & 0.30 \\
\hline \multirow[t]{2}{*}{11} & $\sim 6$ & NS & NS & NS & $\sim 12$ & 0.0 & 0.5 & 0.29 \\
\hline & \multicolumn{4}{|c|}{ pre-SMA versus M1 } & \multicolumn{4}{|c|}{ pre-SMA versus S1 } \\
\hline $\mathrm{Pt}$ & Freq $(\mathrm{Hz})$ & Onset (sec) & Peak (sec) & Peak value & Freq & Onset & Peak & Peak value \\
\hline 1 & - & - & - & - & - & - & - & - \\
\hline 2 & $\sim 12$ & -0.8 & 0.5 & 0.28 & $\sim 12$ & 0.0 & 0.9 & 0.24 \\
\hline 3 & $\sim 18$ & -1.2 & 0.4 & 0.22 & $\sim 12$ & -0.6 & -0.1 & 0.16 \\
\hline 4 & $\sim 12$ & -1.0 & 0.1 & 0.29 & NS & NS & NS & NS \\
\hline 5 & $\sim 6$ & -0.3 & 0.3 & 0.32 & $\sim 6$ & -0.3 & 0.3 & 0.36 \\
\hline 6 & NS & NS & NS & NS & NS & NS & NS & NS \\
\hline 7 & NS & NS & NS & NS & NS & NS & NS & NS \\
\hline 8 & - & - & - & - & - & - & - & - \\
\hline 9 & - & - & - & - & - & - & - & - \\
\hline 10 & - & - & - & - & - & - & - & - \\
\hline \multirow[t]{2}{*}{11} & $\sim 12$ & -0.3 & 0.6 & 0.20 & $\sim 12$ & 0.0 & 0.5 & 0.21 \\
\hline & \multicolumn{4}{|c|}{ M1 versus S1 } & \multicolumn{4}{|c|}{ Pre-SMA versus SMA proper } \\
\hline $\mathrm{Pt}$ & Freq $(\mathrm{Hz})$ & Onset $(\mathrm{sec})$ & Peak (sec) & Peak value & Freq & Onset & Peak & Peak value \\
\hline 1 & $\sim 23$ & -0.8 & 0.5 & 0.36 & - & - & - & - \\
\hline 2 & $\sim 18$ & 0.0 & 0.8 & 0.38 & $\sim 6$ & -0.3 & 0.6 & 0.22 \\
\hline 3 & NS & NS & NS & NS & NS & NS & NS & NS \\
\hline 4 & NS & NS & NS & NS & NS & NS & NS & NS \\
\hline 5 & $\sim 6$ & 0.1 & 0.4 & 0.24 & NS & NS & NS & NS \\
\hline 6 & NS & NS & NS & NS & NS & NS & NS & NS \\
\hline 7 & $\sim 18$ & 0.0 & 0.3 & 0.19 & NS & NS & NS & NS \\
\hline 8 & $\sim 33$ & -0.9 & -0.3 & 0.43 & - & - & - & - \\
\hline 9 & - & - & - & - & - & - & - & - \\
\hline 10 & $\sim 41$ & -0.4 & 0.1 & 0.37 & - & - & - & - \\
\hline 11 & $\sim 23$ & -0.4 & 0.3 & 0.34 & NS & NS & NS & NS \\
\hline
\end{tabular}

Freq, frequency bands showing significant coherence change; NS, no significant change. - , No data available.

$\delta-\theta$ band in four subjects. No subjects showed a maximal coherence increase at frequencies $>20 \mathrm{~Hz}$.

Five subjects (patients 1, 2, 5, 10, and 11) showed near-zero phase lag in the phase spectra, and the remaining two had inconsistent results. The phase difference in the frequency band showing maximal coherence increase was $0 \pm 3^{\circ}$ with respect to $\mathrm{M} 1$.

\section{pre-SMA versus SMA proper}

Coherence between the pre-SMA and SMA proper was analyzed in seven subjects (patients 2-7 and 11). Among them, significant coherence increase was observed in only one subject (patient 2) in $\delta-\theta$ band. Phase spectra revealed near-zero phase lag in this frequency band.

Comparison of the peak value using ANOVA revealed no statistically significant difference for either mesial $(p=0.85)$ or lateral $(p=0.42)$ cortices.

\section{DISCUSSION}

In the present study using event-related partial coherence analysis, we have shown an increase of functional coupling between mesial and lateral frontoparietal cortices starting in the preparatory phase of voluntary hand movements. Extending our previous study (Ohara et al., 2000a), we confirmed the power suppression of mesial and lateral frontoparietal areas during the preparatory phase of the movement. This power suppression of cortical oscillatory activity is thought to reflect cortical activation (Pfurtscheller, 1992). The present study demonstrated (1) that coherence change was preceded by power change by a significant length of time (0.9 sec for coherence between the SMA proper and M1 vs $1.8 \mathrm{sec}$ for the power change in the SMA proper), (2) that the spatial distribution of coherence and power was not significantly correlated, and (3) that the frequency bands showing maximal MPowD and co- 
(A)

\section{coherence}

$\Delta \tanh ^{-1}\left|R_{x y z}(j)\right| 0-6$

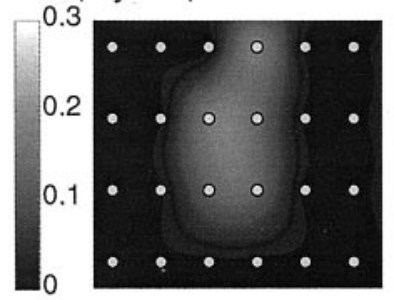

power

$\Delta \log _{10}\left(f_{x x}(i)\right)$

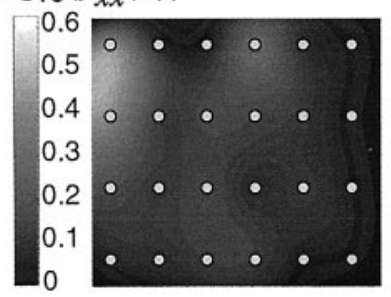

$8-12$

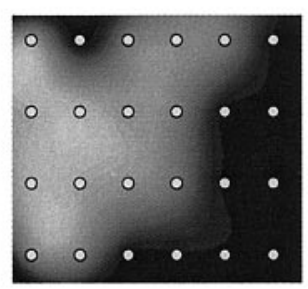

$14-18$

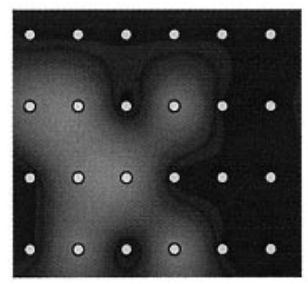

(B)

Figure 4. A, The spatial distribution of coherence increase with respect to the SMA proper in patient 6 . Peak value was plotted as a function of electrode. For the frequency band of $8-12 \mathrm{~Hz}$, coherence peak was present in the postcentral area, whereas for that of $20-23 \mathrm{~Hz}$ it was seen in the precentral area. The spatial distribution of maximal MPowD showed a similar pattern. $B$, Schematic drawing of electrode location including upper extremity $(U / E)$ motor and sensory areas defined by cortical stimulation and the central and precentral sulci determined by 3-D MRI.

(A)

coherence

$\Delta \tanh ^{-1}\left|R_{x y z}(j)\right| 0-6$

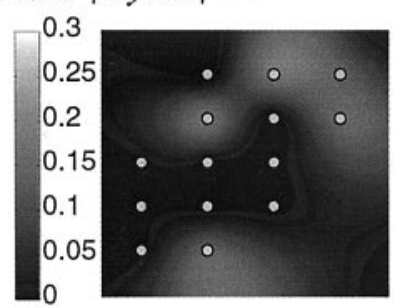

\section{power}

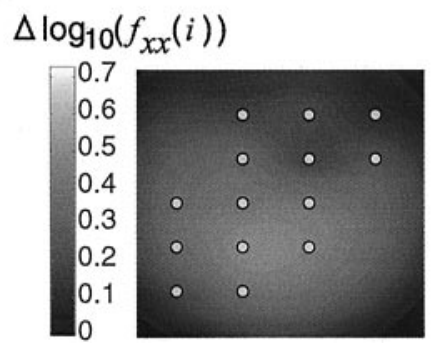

8-12
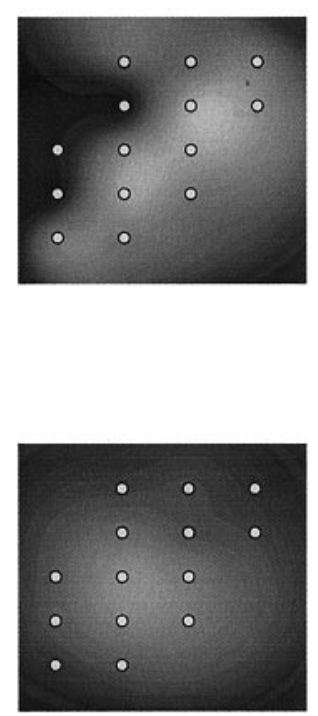
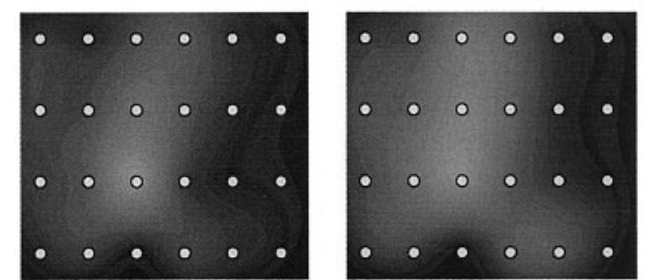

$20-23(\mathrm{~Hz})$
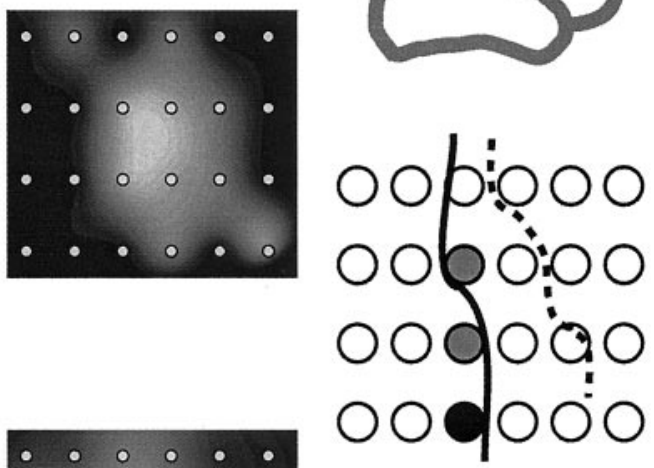

motor U/E sensory U/E central sulcus

.... precentral sulcus

Figure 5. A, The spatial distribution of coherence increase with respect to the pre-SMA in patient 3 . The peak was located in the precentral area. The location of maximal MPowD moved from postcentral to precentral area as a function of frequency. $B$, Schematic drawing of electrode location including upper extremity $(U / E)$ motor area defined by cortical stimulation and the central sulcus.

herence change were different. Thus, it is likely that the movementrelated coherence analysis specifically detects the functional linkage between motor areas, independent of the activation of each area measured by the power change. Furthermore, this method
(B)
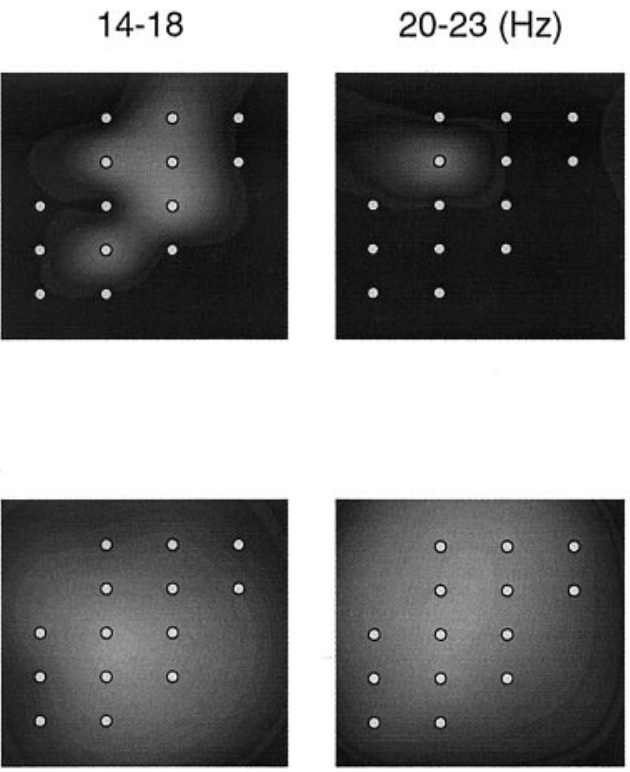

motor U/E

central sulcus demonstrates the temporal change of functional coupling with much higher temporal and spatial resolution than task-related coherence analysis of the scalp-recorded EEG (Classen et al., 1998; Gerloff et al., 1998; Andres et al., 1999). 


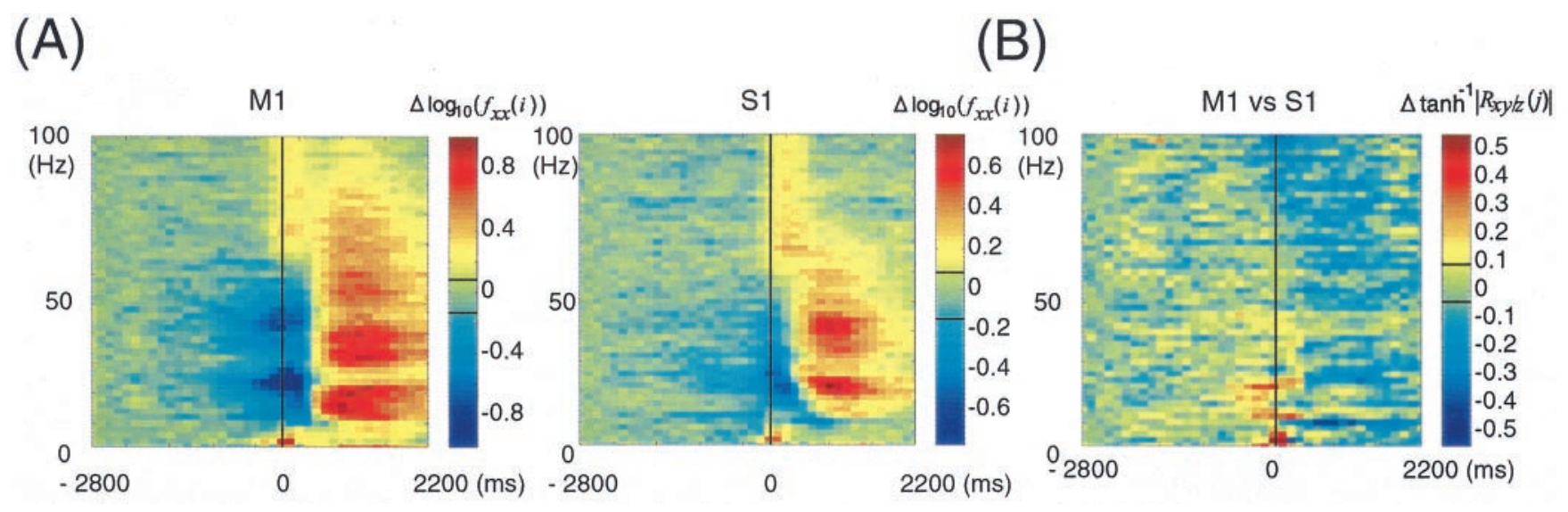

Figure 6. Time-frequency map for power change in M1 and $\mathrm{S} 1(A)$ and for coherence change between those two areas $(B)$ in patient 10 . Note MPowD and subsequent MPowI in the frequency range of $10-60 \mathrm{~Hz}$ and MPowI around the movement onset in the frequency of $>60 \mathrm{~Hz}$. Significant coherence increase was observed in the frequency of up to $\sim 50 \mathrm{~Hz}$ around the movement onset. The horizontal bars in color scale indicate the $95 \%$ confidence limits.

\section{Spectral structure of coherence}

Significant coherence increase was documented in the frequency band $<30 \mathrm{~Hz}$ between mesial and lateral frontoparietal cortices in all subjects except for one (patient 11). Among them, $\beta$ band $(\beta 1$ and $\beta 2)$ activity or even higher frequency activity was involved in half of the analyzed region pairs between mesial and lateral cortices (13 of 27). When the largest coherence increase was taken into account, however, the majority were either in the $\alpha(56 \% ; 15$ of 27$)$ or $\delta-\theta$ bands ( $37 \%$; 10 of 27$)$. Thus, our findings suggest a possible role for low-frequency activity in functional coupling. Low-frequency oscillations have been reported in corticothalamic networks (Steriade et al., 1993a,b) and hippocampus (Vanderwolf, 1969). It was proposed that $\alpha$ and $\theta$ oscillations might reflect cognitive and memory performance (Klimesch, 1999). However, a further study is needed to address the function of the low-frequency coherence. Post-motion components of MRCP (Shibasaki et al., 1980) may affect the lower frequency $(\delta-\theta)$ coherence increase, because of their rhythmic nature in this frequency range. The fact that its onset precedes the movement may exclude the possibility of this apparent coherence change, because the slow rhythmic components of MRCP are particularly seen after the movement onset (Shibasaki et al., 1980).

$\gamma$ band oscillation is thought to be important for cortico-cortical functional coupling (Singer, 1993). In the present study, $\gamma$ oscillation was recorded in M1 and S1, as shown in the previous studies (Crone et al., 1999b; Ohara et al., 2000), but it was rarely seen in the SMA (Figs. 1A,6). However, even between S1 and M1, both of which had abundant $\gamma$ oscillations up to $90 \mathrm{~Hz}$, significant coherence increase in the frequency range of 30-60 $\mathrm{Hz}$ was present in only three subjects (patients 8, 10, and 11) (Fig. 6). Even in these three subjects, significant coherence increase coincided with MPowD at the same frequency band. No coherence increase was observed at the time of MPowI, which followed MPowD. Furthermore, no significant coherence in the high $\gamma$ band $(>60 \mathrm{~Hz})$ was found. Thus, MPowI, which is the local increase of synchrony, might not necessarily be associated with the long-range cortico-cortical functional coupling in voluntary movements. This might support the notion that MPowI after MPowD might represent the deactivated state of the motor cortex (Pfurtscheller and Lopes da Silva, 1999). However, the present findings do not exclude the possibility that $\gamma$ band coherence is involved in other types of tasks (Rodriguez et al., 1999).

\section{Spatial distribution of coherent activity and its functional relevance}

No significant difference in the time course and value of coherence was observed between S1 and M1 (with the SMA proper or the pre-SMA) or between the SMA proper and the pre-SMA (with S1 or M1). Furthermore, the maximal coherence increase with the SMA proper or the pre-SMA was located at either M1, S1, or both, depending on the subjects. This might suggest that the mesial and lateral motor-related areas are linked together as a whole, irrespective of their precise functional properties. This suggests that the motor-related brain areas work as a global network, not as independent components, during movement preparation.

Theoretically, the coherence between mesial and lateral cortices may be explained by a common subcortical oscillator. However, the partial coherence analysis that we used in the present study may exclude its influence, if any, projecting on a wide cortical area. As shown in Figures 4 and 5, the coherence increase was observed in a relatively confined area, which would not be the case if a subcortical oscillator was the main source of coherence. Furthermore, if the subcortical oscillator acts before movement, a power increase should coincide with a coherence increase, contrary to the present result. Thus, it is most likely that the coherence increase observed in the present study might reflect corticocortical connections.

From the anatomical point of view, a tight reciprocal corticocortical connection is present between the SMA proper and M1/premotor cortices (Dum and Strick, 1991; Luppino et al., 1993) and between the SMA proper and S1 (Krubitzer and Kaas, 1990) in primates. However, no direct anatomical connection between the pre-SMA and S1-M1 has been found (Rizzollatti et al., 1998), suggesting that not only cortico-cortical but also corticosubcortical networks (Contreras et al., 1996) might be important for the generation of coherence. However, the definition of preSMA in the present study was based on either the anatomical location with respect to the VAC line or the response to cortical stimulation. Therefore, the pre-SMA in these subjects might include a part of the SMA proper, especially when it was close to the VAC line, which may obscure the difference between them. 


\section{Temporal aspect of functional coupling}

The phase spectra revealed no significant phase difference in 57\% (20 of 35) of region pairs analyzed, and the remaining pairs showed inconsistent results. Thus, it is likely that the motorrelated cortical areas bind together with near-zero time lag in the preparatory phase of voluntary movements. The near-zero phase lag might be in favor of the notion that coherent oscillations could be generated by the common projection from subcortical structures to both medial and lateral cortices. On the other hand, it has been suggested that the near-zero lag among neurons in different cortical areas mediated by reciprocal cortico-cortical connections could represent their integration into a coherent representation (Engel et al., 1991; Munk et al., 1995; Roelfsema et al., 1997).

By using the event-related coherence analysis, we clearly demonstrated that the oscillations recorded from the supplementary and primary motor areas showed increased correlation before voluntary movements. It is postulated that human motor behavior, even a simple voluntary movement, is a product of the complex network connecting the multiple motor-related areas.

\section{REFERENCES}

Allison T, McCarthy G, Wood CC, Jones SJ (1991) Potentials evoked in human and monkey cerebral cortex by stimulation of the median nerve. A review of scalp and intracranial potential. Brain 114:2465-2503.

Andres FG, Mima T, Schulman AE, Dichgans J, Hallett M, Gerloff C (1999) Functional coupling of human cortical sensorimotor areas during bimanual skill acquisition. Brain 122:855-870.

Andrew C, Pfurtscheller G (1995) Event-related coherence during finger movement: a pilot study. Biomed Tech (Berl) 40:326-332.

Andrew C, Pfurtscheller G (1996) Dependence of coherence measurements on EEG derivation type. Med Biol Eng Comput 34:232-238.

Brown P, Salenius S, Rothwell JC, Hari R (1998) Cortical correlate of the Piper rhythm in humans. J Neurophysiol 80:2911-2917.

Classen J, Gerloff C, Honda M, Hallett M (1998) Integrative visuomotor behavior is associated with interregionally coherent oscillations in the human brain. J Neurophysiol 79:1567-1573.

Contreras D, Destexhe A, Sejnowski TJ, Steriade M (1996) Control of spatiotemporal coherence of a thalamic oscillation by corticothalamic feedback. Science 274:771-774.

Conway BA, Halliday DM, Farmer SF, Shahani U, Maas P, Weir AI, Rosenberg JR (1995) Synchronization between motor cortex and spinal motoneuronal pool during the performance of a maintained motor task in man. J Physiol (Lond) 489:917-924.

Cramer SC, Nelles G, Benson RR, Kaplan JD, Parker RA, Kwong KK, Kennedy DN, Finklestein SP, Rosen BR (1997) A functional MRI study of subjects recovered from hemiparetic stroke. Stroke 28:2518-2527.

Crone NE, Miglioretti DL, Gordon B, Sieracki JM, Wilson MT, Uematsu S, Lesser RP (1999a). Functional mapping of human sensorimotor cortex with electrocorticographic spectral analysis. I. Alpha and beta event-related desynchronization. Brain 121:2271-2299.

Crone NE, Miglioretti DL, Gordon B, Lesser RP (1999b) Functional mapping of human sensorimotor cortex with electrocorticographic spectral analysis. II. Event-related synchronization in the gamma band. Brain 121:2301-2315.

Deiber MP, Ibañez V, Sadato N, Hallett M (1996) Cerebral structures participating in motor preparation in humans: a positron emission tomography study. J Neurophysiol 75:233-247.

Donoghue JP, Sanes JN, Hatsopoulos NG, Gáal G (1998) Neural discharge and local field potential oscillations in primate motor cortex during voluntary movements. J Neurophysiol 79:159-173.

Dum RP, Strick PL (1991) The origin of corticospinal projections from the premotor areas in the frontal lobe. J Neurosci 11:667-689.

Eckhorn R, Bauer R, Jordan W, Brosch M, Kruse W, Munk M, Reitboeck HJ (1988) Coherent oscillations: a mechanism of feature linking in the visual cortex? Multiple electrode and correlation analyses in the cat. Biol Cybern 60:121-130.

Engel AK, König P, Kreiter AK, Singer W (1991) Interhemispheric synchronization of oscillatory neuronal responses in cat visual cortex. Science 252:1177-1179.

Freeman WJ (1972) Measurement of oscillatory responses to electrical stimulation in olfactory bulb of cat. J Neurophysiol 35:762-779.

Fried I, Katz A, McCarthy G, Sass KJ, Williamson P, Spencer SS, Spencer DD (1991). Functional organization of human supplementary motor cortex studied by electrical stimulation J Neurosci 11:3656-3666.

Gerloff C, Richard J, Hadley J, Schulman AE, Honda M, Hallett M (1998) Functional coupling and regional activation of human cortical motor areas during simple, internally paced and externally paced finger movements. Brain 121:1513-1531.

Gray CM, Singer W (1989) Stimulus-specific neuronal oscillations in orientation columns of cat visual cortex. Proc Natl Acad Sci USA 86:1698-1702.

Halliday DM, Rosenberg JR, Amjad AM, Breeze P, Conway BA, Farmer SF (1995) A framework for the analysis of mixed time series/point process data-theory and application to the study of physiological tremor, single motor unit discharges and electromyograms. Prog Biophys Mol Biol 64:237-278.

Halliday DM, Conway BA, Farmer SF, Rosenberg JR (1998) Using electroencephalography to study functional coupling between cortical activity and electromyograms during voluntary contractions in humans. Neurosci Lett 241:5-8.

Ikeda A, Lüders HO, Burgess RC, Shibasaki H (1992) Movementrelated potentials recorded from supplementary motor area and primary motor area. Role of supplementary motor area in voluntary movements. Brain 115:1017-1043.

Ikeda A, Lüders HO, Shibasaki H, Collura TF, Burgess RC, Morris III HH, Hamano T (1995) Movement-related potentials associated with bilateral simultaneous, unilateral movements recorded from human supplementary motor area. Electroencephalogr Clin Neurophysiol 95:323-334.

Ikeda A, Lüders HO, Collura TF, Burgess RC, Morris HH, Hamano T, Shibasaki H (1996) Subdural potentials at orbitofrontal, mesial prefrontal areas accompanying anticipation, decision making in humans: a comparison with Bereitschaftspotential. Electroencephalogr Clin Neurophysiol 98:206-212.

Ikeda A, Taki W, Kunieda T, Terada K, Mikuni N, Nagamine T, Yazawa S, Ohara S, Hori T, Kaji R, Kimura J, Shibasaki H (1999a) Focal icta direct current shifts in human epilepsy as studied by subdural and scalp recording. Brain 122:827-838.

Ikeda A, Yazawa S, Kunieda T, Ohara S, Terada K, Mikuni N, Nagamine T, Taki W, Kimura J, Shibasaki H (1999b) Cognitive motor control in human pre-supplementary motor area studied by subdural recording of discrimination/selection-related potentials. Brain 122:915-931.

Klimesch W (1999) EEG alpha and theta oscillations reflect cognitive and memory performance: a review and analysis. Brain Res Brain Res Rev 29:169-195.

Krubitzer LA, Kaas JH (1990) The organization and connections of somatosensory cortex in marmosets. J Neurosci 10:952-974.

Kunieda T, Ikeda A, Ohara S, Yazawa S, Nagamine T, Taki W, Hashimoto N, Shibasaki H (2000) Different activation of presupplementary motor area, supplementary motor area proper, primary sensorimotor area, depending on the movement repetition rate in humans. Exp Brain Res 135:163-172.

Laplane D, Talairach J, Meininger V, Bancaud J, Orgogozo JM (1977) Clinical consequences of corticectomies involving the supplementary motor area in man. J Neurol Sci 34:301-314.

Leocani L, Toro C, Manganotti P, Zhuang P, Hallett M (1997) Eventrelated coherence and event-related desynchronization/synchronization in the $10 \mathrm{~Hz}$ and $20 \mathrm{~Hz}$ EEG during self-paced movements. Electroencephalogr Clin Neurophysiol 104:199-206.

Lesser RP, Gordon B, Fisher R, Hart J, Uematsu S (1992) Subdural grid electrodes in surgery of epilepsy. In: Epilepsy surgery (Lüders HO, ed) pp 399-408. New York: Raven.

Lim SH, Dinner DS, Pillay PK, Lüders H, Morris HH, Klem G, Wyllie E, Awad IA (1994). Functional anatomy of the human supplementary motor area: results of extraoperative electrical stimulation. Electroencephalogr Clin Neurophysiol 91:179-193.

Lüders H, Lesser RP, Dinner DS, Morris HH, Hahn JH, Friedman L, Skipper G, Wyllie E, Friedman D (1987a) Commentary: chronic intracranial recording and stimulation with subdural electrodes. In: Sur gical treatment of the epilepsies (Engel JJ, ed), pp 297-321. New York Raven.

Lüders H, Lesser RP, Morris HH, Dinner DS, Hahn JH (1987b) Negative motor responses elicited by stimulation of the human cortex. Adv Epileptol 16:229-231.

Lüders HO, Lesser RP, Dinner DS, Morris HH, Wyllie E, Godoy J, Hahn JH (1992) A negative motor response elicited by electrical stimulation of the human frontal cortex. Adv Neurol 57:149-157.

Luppino G, Matelli M, Camarda R, Rizzolatti G (1993) Corticocortical connections of area F3 (SMA-proper) and area F6 (pre-SMA) in the macaque monkey. J Comp Neurol 338:114-140.

Mima T, Hallett M (1999) Electroencephalographic analysis of corticomuscular coherence: reference effect, volume conduction and generator mechanism. Clin Neurophysiol 110:1892-1899.

Mima T, Simpkins N, Oluwatimilehin T, Hallett M (1999) Force level modulates human cortical oscillatory activities. Neurosci Lett 275:77-80.

Mima T, Matsuoka T, Hallett M (2000) Functional coupling of human right and left cortical motor areas demonstrated with partial coherence analysis. Neurosci Lett 287:93-96.

Munk MHJ, Nowak LG, Nelson JI, Bullier J (1995) Structural basis of 
cortical synchronization II. Effects of cortical lesions. J Neurophysiol 74:2401-2414.

Murthy VN, Fetz EE (1992) Coherent 25- to 35-Hz oscillations in the sensorimotor cortex of awake behaving monkeys. Proc Natl Acad Sci USA 89:5670-5674.

Murthy VN, Fetz EE (1996) Oscillatory activity in sensorimotor cortex of awake monkeys: synchronization of local field potentials and relation to behavior. J Neurophysiol 76:3949-3967.

Nagamine T, Kajola M, Salmelin R, Shibasaki H, Hari R (1996) Movement-related slow cortical magnetic fields and changes of spontaneous MEG- and EEG-brain rhythms. Electroencephalogr Clin Neurophysiol 99:274-286.

Ohara S, Ikeda A, Kunieda T, Yazawa S, Baba K, Nagamine T, Taki W, Hashimoto N, Mihara T, Shibasaki H (2000a) Movement-related change of electrocorticographic activity in human supplementary motor area proper. Brain 123:1203-1215.

Ohara S, Nagamine T, Ikeda A, Kunieda T, Matsumoto R, Taki W, Hashimoto N, Baba K, Mihara T, Salenius S, Shibasaki H (2000b) Electrocorticogram-electromyogram coherence during isometric contraction of hand muscle in human. Clin Neurophysiol 111:2014-2024.

Pantev C, Makeig S, Hoke M, Galambos R, Hampson S, Gallen C (1991) Human auditory evoked gamma-band magnetic fields. Proc Natl Acad Sci USA 88:8996-9000.

Pfurtscheller G (1992) Event-related synchronization (ERS): an electrophysiological correlate of cortical areas at rest. Electroencephalogr Clin Neurophysiol 83:62-69.

Pfurtscheller G, Aranibar A (1979) Evaluation of event-related desynchronization (ERD) preceding and following voluntary self-paced movement. Electroencephalogr Clin Neurophysiol 46:138-146.

Pfurtscheller G, Lopes da Silva FH (1999) Event-related EEG/MEG synchronization and desynchronization: basic principles. Clin Neurophysiol 110:1842-1857.

Picard N, Strick PL (1996) Motor areas of the medial wall: a review of their location and functional activation. Cereb Cortex 6:342-353.

Rappelsberger P, Pfurtscheller G, Filz O (1994) Calculation of eventrelated coherence-a new method to study short-lasting coupling between brain areas. Brain Topogr 7:121-127.

Rizzolatti G, Luppino G, Matelli M (1998) The organization of the cortical motor system: new concepts. Electroencephalogr Clin Neurophysiol 106:283-296.

Rodriguez E, George N, Lachaux JP, Martinerie J, Renault B, Varela FJ (1999) Perception's shadow: long-distance synchronization of human brain activity. Nature 397:430-433.

Roelfsema PR, Engel AK, König P, Singer W (1997) Visuomotor integration is associated with zero time-lag synchronization among cortical areas. Nature 385:157-161.

Rostomily RC, Berger MS, Ojemann GA, Lettich E (1991) Postoperative deficits and functional recovery following removal of tumors involving the dominant hemisphere supplementary motor area. J Neurosurg 75:62-68.

Salenius S, Salmelin R, Neuper C, Pfurtscheller G, Hari R (1996) Hu- man cortical $40 \mathrm{~Hz}$ rhythm is closely related to EMG rhythmicity. Neurosci Lett 213:75-78.

Salenius S, Portin K, Kajola M, Salmelin R, Hari R (1997) Cortical control of human motoneuron firing during isometric contraction. $\mathrm{J}$ Neurophysiol 77:3401-3405.

Sanes JN, Donoghue JP (1993) Oscillations in local field potentials of the primate motor cortex during voluntary movement. Proc Natl Acad Sci USA 90:4470-4474.

Seitz RJ, Hoflich P, Binkofski F, Tellmann L, Herzog H, Freund HJ (1998) Role of the premotor cortex in recovery from middle cerebral artery infarction. Arch Neurol 55:1081-1088.

Shibasaki H, Barrett G, Halliday E, Halliday AM (1980) Components of the movement-related cortical potential and their scalp topography. Electroencephalogr Clin Neurophysiol 49:213-226.

Shibasaki H, Sadato N, Lyshkow H, Yonekura Y, Honda M, Nagamine T Suwazono S, Magata Y, Ikeda A, Miyazaki M, Fukuyama H, Asato R, Konishi J (1993) Both primary motor cortex and supplementary motor area play an important role in complex finger movement. Brain 116:1387-1398

Singer W (1993) Synchronization of cortical activity and its putative role in information processing and learning. Annu Rev Physiol 55:349-374

Singer W, Gray CM (1995) Visual feature integration and the temporal correlation hypothesis. Annu Rev Neurosci 18:555-586.

Steriade M, McCormick DA, Sejnowski TJ (1993a) Thalamocortical oscillations in the sleeping and aroused brain. Science 262:679-685.

Steriade M, Nunez A, Amzica F (1993b) Intracellular analysis of relations between the slow $(<1 \mathrm{~Hz})$ neocortical oscillation, other sleep rhythms of the electroencephalogram J Neurosci 13:3266-3283.

Tanji J, Kurata K ( 1982) Comparison of movement-related activity in two cortical motor areas of primates. J Neurophysiol 48:633-653.

Toro C, Deuschl G, Thatcher R, Sato S, Kufta C, Hallett M (1995) Event-related desynchronization, movement-related cortical potentials on the ECoG and EEG. Electroencephalogr Clin Neurophysiol 93:380-389.

Vanderwolf $\mathrm{CH}$ (1969) Hippocampal electrical activity and voluntary movement in the rat. Electroencephalogr Clin Neurophysiol 26:407-418.

White LE, Andrews TJ, Hulette C, Richards A, Groelle M, Paydarfar J, Purves D (1997) Structure of the human sensorimotor system: morphology, cytoarchitecture of the central sulcus. Cereb Cortex 7:18-30.

Wise SP, Fried I, Olivier A, Paus T, Rizzolatti G, Zilles KJ (1996) Workshop on the anatomic definition, boundaries of the supplementary sensorimotor area. Adv Neurol 70:489-495.

Zilles K, Schlaug G, Matelli M, Luppino G, Schleucher A, Qu M, Dabringhaus A, Seitz R, Roland P (1995) Mapping of human and macaque sensorimotor areas by integrating architectic, transmitter receptor, MRI and PET data. J Anat 187:515-537.

Zilles K, Schlaug G, Geyer S, Luppino G, Matelli M, Qu M, Schleicher A, Schormann T (1996). Anatomy, transmitter receptors of the supplementary motor areas in the human, nonhuman primate brain. Adv Neurol 70:29-43. 